\title{
Low Velocity Ionized Winds from Regions Around Young O Stars
}

\author{
D.T. Jaffe \\ Department of Astronomy, University of Texas at Austin \\ J. Martín-Pintado \\ Instituto Geográfico Nacional, Observatorio Astronómico Nacional
}

Received — 


\begin{abstract}
We have observed seven ultracompact H II regions in hydrogen recombination lines in the millimeter band. Toward four of these regions, there is a high velocity (full width to half maximum $60-80 \mathrm{~km} \mathrm{~s}^{-1}$ ) component in the line profiles. The high velocity gas accounts for $35 \%-70 \%$ of the emission measure within the beam. We compare these objects to an additional seven similar sources we have found in the literature. The broad recombination line objects (BRLOs) make up about $30 \%$ of all sources in complexes containing ultracompact H II regions. Comparison of spectral line and continuum data implies that the BRLOs coincide with sources with rising spectral indices, $\geq 0.4$ up to $100 \mathrm{GHz}$. Both the number of BRLOs and their frequency of occurrence within H II region complexes, when coupled with their small size and large internal motions, mean that the apparent contradiction between the dynamical and the population lifetimes for BRLOs is even more severe than for ultracompact $\mathrm{H}$ II regions as a whole. We evaluate a number of possible models for the origin of the broad recombination line emission. The lifetime, morphology, and rising spectral index of the sources argue for photoevaporated disks as the cause for BRLOs. Existing models for such regions, however, do not account for the large amounts of ionized gas observed at supersonic velocities.
\end{abstract}

Subject headings: ISM:H II Regions ISM:Jets and Outflows Radio Lines:Stars Stars:Mass Loss 


\section{Introduction}

The formation, behavior, and evolution of $\mathrm{O}$ stars influence many aspects of the interstellar medium in the Galaxy. O stars play a central role in the evolution of galactic

abundances, influence the structure of the interstellar medium through supernova explosions, ionization, and mass-loss, and determine the appearance of the spiral arms in the visible and the radio through both thermal and non-thermal emission. The pre-main sequence evolution of luminous $\mathrm{O}$ stars takes place while they are still shrouded within their natal clouds. Therefore, we usually study the surrounding H II regions and dust clouds, rather than the stars themselves, and carry out these studies at far-IR and radio wavelengths.

Much of the gas in the H II regions that surround newly formed O stars is involved in supersonic motions of the order of $20 \mathrm{~km} \mathrm{~s}^{-1}$, usually ascribed to "turbulence". The smallest and presumably youngest H II regions (ultracompact, or UC H II regions) typically have linewidths of $25-35 \mathrm{~km} \mathrm{~s}^{-1}$ in high frequency centimeter wave recombination lines (RRL's, Garay 1989, Churchwell et al. 1989, Afflerbach et al. 1996). There is considerable observational evidence for even higher velocity motions of various sorts. Massive O stars have winds with terminal velocities of $1-2 \times 10^{3} \mathrm{~km} \mathrm{~s}^{-1}$ and mass loss rates of up to a few $10^{-6} \mathrm{M}_{\odot}$ per year (Chlebowski \& Garmany 1991). Many high luminosity and presumably young stars embedded deep within molecular clouds show evidence for ionized circumstellar envelopes or for winds with terminal velocities $\sim 200 \mathrm{~km} \mathrm{~s}^{-1}$ (Simon et al. 1981, 1983, Persson et al. 1984, Garden \& Geballe 1986, Bunn, Hoare, \& Drew 1995). In some more evolved and optically accessible H II regions, there are small amounts of gas moving at 100-200 $\mathrm{km} \mathrm{s}^{-1}$ with respect to the bulk velocity of the H II region (Meaburn \& Walsh $1981,1986)$.

There is increasing evidence that a (substantial) minority of ultracompact H II regions emit broad radio recombination lines. (In this paper, we refer to these sources as broad 
recombination line objects, BRLOs). The presence of this RRL component implies that the

regions contain gas moving at velocities up to $\pm 60 \mathrm{~km} \mathrm{~s}^{-1}$ from the rest velocity of the cloud (Altenhoff, Strittmatter, \& Wendker 1981, Zijlstra et al. 1990, Gaume, Fey, \& Claussen 1994, De Pree et al. 1994, 1996). In the UC H II regions, unlike the more evolved H II regions, the high velocity gas may account for a significant fraction of the total emission measure. If high velocity emission arises in the very compact parts of the UC H II region complexes where the emission measures are high, the gas may only become detectable in observations at the shortest centimeter wavelengths where these compact sources begin to become optically thin and where non-LTE effects become negligible. Observations at shorter wavelengths could therefore provide a better way to search for the broad features. We have investigated the incidence and nature of the broad recombination line features by surveying a sample of bright UC H II regions in 3,2 , and $1.3 \mathrm{~mm}$ recombination lines. This paper presents results and analysis of this survey and a discussion of the origin of the broad line emission.

\section{Observations}

We made all the observations we present here with the IRAM 30 meter telescope at Pico de Veleta (Spain). The first set of observations took place in 1992 February and the second set in 1993 March. On the fist observing run, we observed three recombination lines simultaneously at wavelengths of 3,2 and $1.3 \mathrm{~mm}$, and on the second run, we observed several combinations of two such lines (see Table 1). The telescope beam at these wavelengths was, respectively, 24,17 and 11 arc seconds. Table 1 lists the sources, source positions, dates of observations, and observed transitions. The receivers were SIS mixers, tuned to single sideband (image rejections between 7 and $10 \mathrm{~dB}$ ), with typical system noise temperatures of 250, 350 and $650 \mathrm{~K}$ at 3,2 and $1.3 \mathrm{~mm}$. We used two 512 
channel filterbanks with $1 \mathrm{MHz}$ resolution and a 430 channel Acousto-Optical Spectrometer with a resolution of $1.1 \mathrm{MHz}$ as spectrometric backends. In order to insure good baseline subtraction, we nutated the telescope subreflector at $0.25 \mathrm{~Hz}$ between the source and a reference position 240 arc seconds away. While some of the sources have extended radio continuum emission, published maps at centimeter wavelengths show that an angular displacement of this size should result in contamination of the reference beam by narrow recombination line emission at only a very low level. Under good weather conditions, use of the nutating subreflector allows one to measure the line and continuum emission simultaneously. The weather conditions during the 1993 observations were good enough to permit us to measure the continuum emission at $3 \mathrm{~mm}$. We calibrated the data by observing the atmosphere and cold and hot loads at known temperatures. All the intensities are in units of antenna temperature corrected for forward scattering and spillover as well as for atmospheric transmission, $\mathrm{T}_{A}^{*}$, and the conversion factor from antenna temperature to flux density at $3 \mathrm{~mm}$ is $6 \mathrm{Jy} / \mathrm{K}$.

Almost all of the ultracompact H II regions studied as part of the present work lie close to or within dense, high column density molecular cores. These cores produce very rich millimeter wave line spectra. Confusion from these molecular line emitters within the beam is an important limiting factor when deriving quantitative results from the millimeter recombination line data. The presence of the molecular lines does not, however, impede our ability to detect high velocity recombination line emission. The molecular lines are, in general, quite narrow (5-8 $\mathrm{km} \mathrm{s}^{-1}$ ) and the broad components of adjacent $\Delta \mathrm{n}=1$ recombination transitions with similar $\mathrm{n}$ should be similar in strength and shape but overlap with completely different sets of molecular features. 


\section{Results and Analysis}

Table 2 presents the best fits of one or two Gaussians to profiles of selected lines in each source in our survey. In all cases, the lines selected from among the $1.3-3 \mathrm{~mm}$ Hn $\alpha$ transitions observed (Table 1) are the $3 \mathrm{~mm}$ transitions, H39 $\alpha$ and H41 $\alpha$. Since our observed positions coincide with or lie close to hot, dense, high column density molecular cores (cf. Plume, Jaffe, \& Evans 1992, Plume et al. 1997), the high density of molecular features at $2 \mathrm{~mm}$ and $1.3 \mathrm{~mm}$ makes the hydrogen recombination lines in these bands more prone to confusion. The $3 \mathrm{~mm}$ continuum flux densities measured toward the positions given in Table 1 also appear in Table 2. Table 2 gives the electron temperatures, derived from the ratio of the total integrated $\mathrm{H} 41 \alpha$ line intensity (including both line components if more than one is present) to the measured continuum adjacent to this $3 \mathrm{~mm}$ line, assuming LTE conditions. It is interesting to note that failure to notice the presence of a relatively weak broad component in recombination line spectra, a component which can be missed in observations with moderate sensitivity, can lead one to overestimate the electron temperatures derived from the line-to-continuum ratios. The LTE electron temperatures

we derive from our data all lie within $\pm 30 \%$ of the canonical $10,000 \mathrm{~K}$ temperature of H II regions.

\subsection{Broad Recombination Line Objects}

The millimeter recombination line shapes for four of the seven sources in our sample (NGC 7538, W49N, G34.3+0.2, and S106) deviate substantially from the simple 25-35 km $\mathrm{s}^{-1}$ wide Gaussian profiles that are typical of ultracompact H II regions. Fits of single Gaussians to these profiles left systematic residuals at $|V| \geq 20 \mathrm{~km} \mathrm{~s}^{-1}$ from the line center. In all cases, we significantly reduce the residuals when we fit the observed spectra with two Gaussian profiles, one with the normal UC H II region recombination line width and 
another broad component which is $2-3$ times wider. We present here our analysis of these four sources.

Toward most of the sources in our sample, the $30 \mathrm{~m}$ beam contains very complex radio continuum structure that contributes to both the normal and the broad spectral components. So far, there are no maps of recombination lines at millimeter wavelengths with sufficient angular resolution and sensitivity to identify the sources responsible for the broad features. To identify the sources giving rise to these features, we combine our millimeter recombination line data with existing high angular resolution maps of continuum and recombination line emission, mainly observed at centimeter wavelengths. To aid in comparisons with these maps, we estimate the amount of continuum emission "required" to produce each line component by assuming that the observed $3 \mathrm{~mm}$ line and continuum emission is optically thin and that the millimeter recombination lines arise from gas where the electron temperature equals the temperature derived from the total line and continuum emission (under the assumption of LTE conditions) from each source (Table 2). Table 8 gives our estimate of the continuum associated with the broad and the normal components based on these assumptions. The gas that produces the broad line component accounts for $35 \%-70 \%$ of the $3 \mathrm{~mm}$ continuum flux and therefore at least that fraction of the emission measure in these four objects.

\subsection{1. $N G C 7538$}

We have observed six millimeter wave hydrogen recombination lines toward NGC 7538. Our beam center lies at the position of the infrared source and double radio continuum source NGC 7538 IRS1 (Campbell 1984). A free fit of a single Gaussian profile to the H41 $\alpha$ line underestimated the brightness both at the peak of the line and at high velocities. The left panel of Figure 1 shows the results of a fit which we forced to match the area around 
the peak by minimizing the least squares deviation between a single Gaussian profile and the observed line over a range of $\pm 15 \mathrm{~km} \mathrm{~s}^{-1}$ about the peak of the line. The lower part of the left panel shows that, while this profile accounts well for the inner part of the observed line, there are substantial residuals to this fit, in particular at higher velocity. The right panels of Figure 1 show a fit of two Gaussians to the same profile and the residuals resulting from this fit. The line parameters we derive in an independent fit of two Gaussians to the H39 $\alpha$ line agree well with the H41 $\alpha$ results (Table Q). For both hydrogen lines, the normal line has a width of around $25 \mathrm{~km} \mathrm{~s}^{-1}$ while the broad component has a width of $70 \mathrm{~km} \mathrm{~s}^{-1}$.

High resolution $1.3 \mathrm{~cm}$ recombination line observations toward NGC 7538 IRS1 (Gaume et al. 1995) reveal extremely broad emission (full width to zero power of around $200 \mathrm{~km} \mathrm{~s}^{-1}$ in the northern and southern hypercompact lobes and an absence of line emission in between the lobes. The discrepancies between the linewidths of the centimeter and millimeter wavelength recombination lines are similar to those found in MWC 349. As in this latter source, the broad H66 $\alpha$ lines in NGC 7538 IRS1, which show noticeable non-Gaussian profiles with flat-topped shapes, are very likely affected by non-LTE excitation (see Martín-Pintado et al 1993).

For NGC 7538, our beam contains the sources IRS1, IRS2 and IRS3 and the total continuum flux density at $3 \mathrm{~mm}$ is in good agreement with the fluxes derived by Akabane et al. (1992) from interferometric measurements. The $3^{\prime \prime}$ resolution $3 \mathrm{~mm}$ continuum map (Akabane et al. 1992) shows that IRS3 makes a negligible contribution to the millimeter wave continuum flux, IRS2 contributes $1 \mathrm{Jy}$, and IRS1 contributes $1.6 \mathrm{Jy}$. The values for the continuum fluxes for the two latter sources are consistent with an attribution of the normal spectral component to IRS2 and the broad component to IRS1, the same sense in which the origin of the normal and very broad $6 \mathrm{~cm}$ recombination line emission is directly attributable to the respective sources (Gaume et al. 1995). 


\subsection{2. $W 49 N$}

We observed six millimeter wave hydrogen recombination lines toward this source (Table 回). Our beam center lies toward the $\mathrm{H}_{2} \mathrm{O}$ maser center, about $1^{\prime \prime}$ north of the ultracompact $15 \mathrm{GHz}$ continuum source G1 (Dreher et al. 1984). The upper left panel of Figure 2 shows a single Gaussian fit to the H41 $\alpha$ line made following the procedure described for the single Gaussian fit to the NGC 7538 profile. The residuals to this fit (lower left) show a systematic excess in the wings of the line. The upper right panel of Figure 2 shows a two Gaussian fit to the same line. The minimization results in a component with a velocity width of $31 \mathrm{~km} \mathrm{~s}^{-1}$ and a second component $71 \mathrm{~km} \mathrm{~s}^{-1}$ wide. Over the velocity interval occupied by the hydrogen line, the residuals to the two Gaussian fit at the central position are flat. We also fit two Gaussians to the He41 $\alpha$ line at this position. We allowed the temperatures of the two components to vary but fixed the widths of the two He components at the values of the $\mathrm{H} 41 \alpha$ widths and the velocity offset from the corresponding H41 $\alpha$ components at $-122.2 \mathrm{~km} \mathrm{~s}^{-1}$. This fit (Figure 2 (lower right)) shows that a normal plus a broad component can account for the shape of the helium line as well. The strengths of the normal and broad He components are respectively $9 \%$ and $8 \%$ of the strengths of the corresponding hydrogen emission components. A five point map of the H41 $\alpha$ transition at $12^{\prime \prime}$ intervals is consistent with an unresolved source as the origin of both the normal and the broad component.

The upper panel of Figure 3 superposes spectra of $\mathrm{H} 39 \alpha$ and $\mathrm{H} 41 \alpha$ toward W49N. The wings of the two recombination lines match very closely, but the shapes of the inner portions of the lines differ considerably. A triple Gaussian fit to the H39 $\alpha$ spectrum fixing the velocities and widths of the two broader components to match the values derived from the $\mathrm{H} 41 \alpha$ spectrum shows that there is a third feature with a full width to half maximum of $13.3 \mathrm{~km} \mathrm{~s}^{-1}$. This narrower feature has a width similar to the width of molecular lines 
in this source (Serabyn, Guesten \& Schulz 1993). The third line has an observed velocity of $-7.5 \mathrm{~km} \mathrm{~s}^{-1}$ with respect to the $\mathrm{H} 39 \alpha$ frequency. The only molecular transition with a rest frequency close to that of the $\mathrm{H} 39 \alpha$ line $(106737.365 \mathrm{MHz})$ is the $2_{3}-1_{2}$ transition of ${ }^{34} \mathrm{SO}\left(\nu_{o}=106743.365 \mathrm{MHz}\right)$. For this line, the observed velocity corresponds to $\mathrm{V}_{L S R}=6.6$ $\mathrm{km} \mathrm{s}^{-1}$ in its own frame. This velocity is close to the typical molecular velocity of the W49 core. The lower panel of Figure 3 shows an overlay of the observed H39 $\alpha$ and H41 $\alpha$ profiles where we have added the fitted ${ }^{34} \mathrm{SO}$ feature to the H41 $\alpha$ data to illustrate the similarity of the shapes of the recombination line profiles themselves.

There is some evidence for high velocity recombination line emission in previous millimeter observations of W49N. The wings seen in Figure 2 are also apparent to the trained eye in the $\mathrm{H} 40 \alpha$ spectrum in Figure 3 of Gordon (1989). High angular resolution observations of the $\mathrm{H} 66 \alpha$ and $\mathrm{H} 52 \alpha$ transitions at $1.3 \mathrm{~cm}$ and $0.7 \mathrm{~cm}$, respectively (De Pree, Mehringer, \& Goss 1997), show that sources A and B, both of which lie within our beam, produce lines with a full width to half maximum of $50-60 \mathrm{~km} \mathrm{~s}^{-1}$. Since the line fills the spectrometer band for these sources, however, the observations do not exclude the presence of higher velocity emission consistent with our millimeter measurements.

Determining the source or sources in W49N responsible for the broad line emission is complex because of the large number of continuum sources in our beam. The H41 $\alpha$ beam contains the sources A-G2. We can use high angular resolution maps of the RRL and continuum emission (De Pree et al. 1997) to establish which source may be responsible for the broad component. As previously mentioned, sources A and B have the broadest H66 $\alpha$ and $\mathrm{H} 52 \alpha$ profiles. These two sources have power law radio continuum spectra with spectral indexes of 0.6 and 0.9 , respectively. Extrapolating to $3 \mathrm{~mm}, \mathrm{~A}$ and B would each contribute around 2 Jy. Given the 3 Jy required to provide the source of the broad line emission (see Table 2), these two stellar wind sources are viable candidates for the origin of the broad 
lines. However, source $\mathrm{A}$ is located at the edge of the beam and the map of the H39 $\alpha$ line indicates a point source located closer to B than to A. Like in NGC 7538 IRS1, the broad component seems to be associated with compact sources with rising continuum spectra.

\subsection{3. $G 34.3+0.2$}

We observed six millimeter wave hydrogen recombination lines toward this source (Table 1). The center position for these observations lies about $3^{\prime \prime}$ south of the $15 \mathrm{GHz}$ continuum peak (Garay, Rodriguez, \& van Gorkom 1986). The left panel in Figure 4 shows a single Gaussian fit to the H41 $\alpha$ transition toward G34.3+0.2 (see the discussion of NGC 7538 for a description of the fitting procedure). The residuals (lower left) show significant excess emission at high velocities, particularly on the blue side of the line. The right panel of Figure 4 shows a fit using two Gaussians. Superposed on the residuals of this fit is a fit to the helium line made by holding the relative velocity to the corresponding hydrogen component fixed and setting the widths of the two helium components equal to those of the hydrogen components. Figure 5 shows a superposition of the H41 $\alpha$ and H39 $\alpha$ lines toward G34.3+0.2. The figure also shows the sum of two Gaussians with parameters averaged between those from the $\mathrm{H} 41 \alpha$ and $\mathrm{H} 39 \alpha$ line fits. The compromise Gaussians have widths of 32 and $75 \mathrm{~km} \mathrm{~s}^{-1}$. The shape of the two hydrogen lines agree well, except at velocities where narrow molecular lines from the hot core contaminate the profiles.

Toward G34.3+0.2, our $30 \mathrm{~m}$ beam at $3 \mathrm{~mm}$ contains three radio continuum sources. Components $\mathrm{A}$ and $\mathrm{B}$ are compact objects with spectra that rise with frequency at least to $90 \mathrm{GHz}$ (Gaume et al. 1994). Component C is a classic cometary HII region (Reid \& Ho 1985; Garay et al. 1986). At centimeter wavelengths, where sources A and B are weak and opaque, all of the recombination line emission comes from source $\mathrm{C}$. The $2 \mathrm{~cm}$ results (Garay et al. 1986) indicate a relatively uniform line width of around $50 \mathrm{~km} \mathrm{~s}^{-1}$ 
across source C. The more sensitive $3 \mathrm{~cm}$ data (Gaume et al. 1994) show line widths which increase from $25-45 \mathrm{~km} \mathrm{~s}^{-1}$ in the north and west to about $80 \mathrm{~km} \mathrm{~s}^{-1}$ in the southeast of component C (Gaume et al. 1994).

Our total continuum emission measured at $3 \mathrm{~mm}$ is $\sim 7 \mathrm{Jy}$, in good agreement with the 6.6 Jy we extrapolate from the 6 and $2 \mathrm{~cm}$ measurements of Benson \& Johnston (1984) by assuming that source $\mathrm{C}$ is optically thin and ultracompact sources A and B are optically thick with spectral indexes of $\sim 0.9$ (Gaume at al. 1994). Based on this extrapolation, the compact sources A and B, despite their rising "stellar wind" spectra, can only account for $0.8 \mathrm{Jy}$ of the $7 \mathrm{Jy}$ flux density at $3 \mathrm{~mm}$. Both the broad and the narrow line component therefore should arise mainly from the cometary H II region. The radial velocities and widths of both the broad and narrow line components in the H41 $\alpha$ spectrum lie within the range of one or another centimeter RRL measurement toward the parts of source $\mathrm{C}$ that emit most of the flux (Garay et al. 1986, Gaume et al. 1994). The results obtained by the centimeter RRL observers, however, are not consistent with each other.

\subsection{4. $S 106$}

S106 is less luminous than the other BRLOs in our sample. We selected this source for investigation because of the numerous observations of broad near-IR hydrogen recombination lines toward S106 IR (Persson et al. 1984, Felli et al. 1985, Garden \& Geballe 1986, Drew, Bunn, \& Hoare 1993). This IR source lies at the center of an obscuring dust disk (Eiroa, Elsaesser, \& Lahulla 1979) and at the edge of our beam, 13" northeast of the position listed in Table 1. Coincident with the IR source is a compact radio source with a spectrum rising as $\nu^{0.6}$ from 5 to $23 \mathrm{GHz}$ (Snell \& Bally 1986, Felli et al. 1985). Very high angular resolution $5 \mathrm{GHz}$ observations show that this central source is elongated along the equator of the extended bipolar optical and radio nebula (Hoare et al. 1994, Felli 
et al. 1984). There is a strong submillimeter dust continuum source close to the position we observed (Richer et al. 1993). The difference between the observed position and the IR source position and the presence of relatively strong dust emission make modeling this source problematic.

For S106, the broad component cannot arise only from the compact continuum source with the rising spectrum since the extrapolation of the continuum spectrum of this source to $3 \mathrm{~mm}$ implies that it will only account for $42 \mathrm{mJy}$ at this wavelength. Therefore, the high-velocity recombination line emission is associated with the bipolar nebula. Like G34.3+0.2, this source appears to be more evolved than the UC HII regions in NGC 7538 IRS1 and W49N. Long slit spectroscopy in the optical shows that S106 has the velocity structure of a bipolar outflow (Solf \& Carsenty 1982). There is H $\alpha$ emission throughout the southern lobe of the extended bipolar source. $\mathrm{H} \alpha$ has a total extent of over $100 \mathrm{~km} \mathrm{~s}^{-1}$ at the position we observed at $3 \mathrm{~mm}$. Farther south in this lobe, the H $\alpha$ lines split into two components with typical separations of $50 \mathrm{~km} \mathrm{~s}^{-1}$ (Solf \& Carsenty 1982).

\subsection{Narrow Line Sources}

\subsubsection{Orion/IRc2 and Orion $\mathrm{H}_{2}$ Peak 1}

Figure 6 shows an $\mathrm{H} 39 \alpha$ spectrum toward $\mathrm{H}_{2}$ Peak 1 (left) and an $\mathrm{H} 41 \alpha$ spectrum toward IRc2 (right) in Orion. In both cases, the residuals below each spectrum demonstrate that a single Gaussian fits the line profile well. Hasegawa \& Akabane (1984) have reported

high velocity emission toward Peak 1 in the H51 $\alpha$ line. The reported high velocity emission gave rise to the suggestion that such emission could come from gas ionized by the radiative precursor of a dissociative shock in the outflowing molecular gas (McKee \& Hollenbach 1987). Our H39 $\alpha$ observation at the position of Peak 1 excludes the presence of a broad 
component at a level about a factor of five lower than the $0.1 \mathrm{~K}$ implied by the putative H51 $\alpha$ detection. Our H41 $\alpha$ observations toward IRc2 also show no evidence for emission beyond that arising from a single $23 \mathrm{~km} \mathrm{~s}^{-1}$ wide Gaussian component. There is therefore no observational evidence for shock-ionized gas in the region around Orion/IRc2. It would be better to search for recombination lines from shock-ionized gas where little or no confusing photoionized gas is present. Possible targets might include the high velocity shocked regions in powerful outflows associated with less luminous sources like IRAS 03282 and L1448 (Bachiller 1996).

\subsection{2. $G 10.6-0.4$}

G10.6-0.4 is a bright UC HII region situated in a molecular core. Observations of the radio continuum at 6,2 , and $1.3 \mathrm{~cm}$ show several emission knots within 5" (Ho \& Haschick 1981, 1986). We have observed two lines toward this source, H35 $\alpha$ and H41 $\alpha$ (Figure 7). The single Gaussian fit to the less confused H41 $\alpha$ line (Table 2) undershoots the peak of the line and overshoots in the wings. This behavior mimics that of the residuals of single, unconstrained Gaussian fits to the lines in G34.3+0.2 and W49, indicating the possible presence of a broad component. The signal to noise ratio of the G10.6-0.4 spectra, however, is insufficient to warrant a fit of more than one Gaussian.

\subsubsection{DR21}

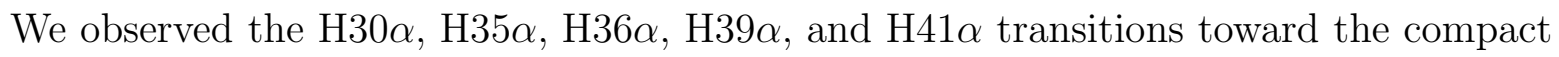
H II region in DR21. Figure 8 shows the H39 $\alpha$ line. The residuals in this figure indicate that a single Gaussiam with a width of $32 \mathrm{~km} \mathrm{~s}^{-1}$ can account for all of the flux in the hydrogen line. This source has strong molecular and neutral atomic emission at high velocities (Bally 
\& Lada 1983, Russell et al. 1992) but does not have a corresponding ionized gas component in the millimeter recombination lines.

\section{Other Broad Recombination Line Objects}

A search of the literature shows that there are at least seven additional ultracompact HII regions with high velocity $\left(\Delta \mathrm{V} \geq 55 \mathrm{~km} \mathrm{~s}^{-1}\right)$ emission in recombination lines at $\mathrm{n} \leq 80$, where pressure broadening is less likely to be important. These sources are: the four sources comprising SgrB2M F, G5.89-0.39, MWC 349, and K3-50A (see Table 2).

As a whole, SgrB2M source F has broad H66 $\alpha$ and H52 $\alpha$ lines (Mehringer et al. 1995, De Pree et al. 1996). Integrating over the source, the H52 $\alpha$ line has a FWHM of $59 \mathrm{~km}$ $\mathrm{s}^{-1}$. The H66 $\alpha$ observations with the VLA resolve source F into four subsources. The line widths for these more compact objects range from $68 \mathrm{~km} \mathrm{~s}^{-1}$ to $79 \mathrm{~km} \mathrm{~s}^{-1}$. Each of the subsources has a rising continuum spectrum at $1 \mathrm{~cm}$.

G5.89-0.39 contains an ultracompact source (Source A) with broad RRL emission. Zijlstra et al. (1990) detected a normal $\left(\Delta \mathrm{V}=22 \mathrm{~km} \mathrm{~s}^{-1}\right)$ and a broad $\left(\Delta \mathrm{V}=64 \mathrm{~km} \mathrm{~s}^{-1}\right)$ H76 $\alpha$ component toward this source. Their continuum measurements imply a spectral index of $\nu^{2.2}$ from $20 \mathrm{~cm}$ to $6 \mathrm{~cm}$ and $\nu^{0.8}$ from $6 \mathrm{~cm}$ to $2 \mathrm{~cm}$. Afflerbach et al. (1996) measured line widths of $59 \mathrm{~km} \mathrm{~s}^{-1}$ and $61 \mathrm{~km} \mathrm{~s}^{-1}$ at $\mathrm{H} 42 \alpha$ and $\mathrm{H} 66 \alpha$, respectively. Their data imply a spectral index of $\nu^{0.55}$ from $1.3 \mathrm{~cm}$ to $3 \mathrm{~mm}$. Additional continuum data in the literature give somewhat inconsistent results (Acord, Churchwell, \& Wood 1997; Acord, Walmsley, \& Churchwell 1998; Wood and Churchwell 1989), but all of the results imply a rising spectrum throughout the centimeter band and continuuing to at least $7 \mathrm{~mm}$. Acord et al. (1998) have used $3.6 \mathrm{~cm}$ continuum observations over a 5 year span to measure what they argue is a proper motion of a shell due to dynamical expansion. 
MWC 349 is an isolated HII region in Cygnus. This object is the archetypal ionized stellar wind source. Its spectrum rises as $\nu^{0.6}$ from $\mathrm{cm}$ wavelengths up to $60 \mu \mathrm{m}$ (Harvey, Thronson, \& Gatley 1979). This behavior is characteristic of an isothermal wind expanding at constant velocity (see e.g. Wright \& Barlow 1975). The typical line widths measured for the centimeter wave recombination lines (Altenhoff et al. 1981) and optical [N II] lines (Hartmann, Jaffe, \& Huchra 1980) is $\sim 60 \mathrm{~km} \mathrm{~s}^{-1}$. VLA observations of the H66 $\alpha$ line show that the lines have nearly flat topped profiles with a width of around $100 \mathrm{~km}$ $\mathrm{s}^{-1}$, substantially larger than those measured from the Gaussian profiles of the $3 \mathrm{~mm}$ recombination lines (Martín-Pintado et al 1989). Modeling of the continuum and the line intensities of the $\mathrm{H} 66 \alpha$ lines indicates that the discrepancies are likely to be due to non-LTE effects in the centimeter recombination lines (see Martín-Pintado et al 1993). MWC 349 shows a bipolar morphology (White \& Becker 1985), but unlike K3-50A (see below), it does not show a bipolar distribution in the recombination lines (Martín-Pintado et al 1993); indicating that MWC 349 must be oriented perpendicular to the line of sight. As an object for study, MWC 349 has the advantage over the other BRLOs detected so far that it is isolated and the ionizing star can be seen in the visible and the near-IR. In some sense, MWC 349 may play the role for massive stars that field TTauri stars play in studies of low mass stars.

At 2-6 cm, the elongated continuum source $\mathrm{K} 3-50 \mathrm{~A}$ dominates the radio continuum emission from compact radio sources in the K3-50 complex. Combining the continuum data of Roelfsema, Goss, \& Geballe (1988) and De Pree et al. (1994), we estimate a 5-15 GHz spectral index for this source of $\nu^{0.5}$. Roelfsma et al. (1988) detected a broad $(\Delta \mathrm{V}=66 \mathrm{~km}$ $\mathrm{s}^{-1}$ ) component to the $\mathrm{H} 110 \alpha$ line but attributed its breadth to pressure broadening. The more recent H76 $\alpha$ results (De Pree et al. 1994) show not only that the large line widths are a dynamical effect but also that there is clear evidence in this source for an ionized bipolar outflow. Toward the center of the source, the $2 \mathrm{~cm}$ recombination line has a width of $76 \mathrm{~km}$ 
$\mathrm{s}^{-1}$. Three arc seconds north and south of this position, the width is only $35 \mathrm{~km} \mathrm{~s}^{-1}$ but the velocity changes from $-11 \mathrm{~km} \mathrm{~s}^{-1}$ north of the peak to $-42 \mathrm{~km} \mathrm{~s}^{-1}$ to the south.

\section{Discussion}

\subsection{Observational Constraints on Possible Models}

In this section, we summarize the observations and key parameters derived from the observations for which any viable model for objects that produce the broad recombination lines (BRLOs) must account.

\subsubsection{Lifetimes}

BRLOs are common in UC H II region complexes. The lifetimes of these sources are much longer than their crossing times and the broad line emission phase must represent a substantial fraction of the lifetime of UC H II sources.

One argument for the long duration of the broad line emission phase as a part of overall H II region evolution is the presence of broad millimeter and high frequency centimeter recombination lines toward ultracompact sources like NGC 7538 IRS1 and MWC 349 and also toward more extended and presumably more evolved sources like K3-50 and S106. It is possible that there is a connection between the dominant broad line emission at early evolutionary stages and the low-level emission at high velocities seen in very evolved HII regions (Meaburn \& Walsh 1981, 1986). The available sample of H II regions observed with high enough sensitivity to detect the broad recombination lines is small (Table 3). As a consequence, our estimate of the fraction of compact H II regions with broad lines is somewhat uncertain and may suffer from selection biases. We follow two approaches in 
estimating the frequency of occurrence of these objects: We first determine the fraction of sources in UC H II region complex that show the broad lines, within each well-studied complex containing at least one BRLO. In NGC 7538, one out of three sources is a BRLO. In G34.3+0.2 one to three out of three is likely to be a BRLO and in W49, we find that two out of eight sources are probably responsible for the broad recombination line emission. From these data, we estimate that roughly around $30 \%$ of the compact sources in these complexes are BRLOs.

As a second approach, we use a limited sample of compact H II regions (Afflerbach et al. 1996). Since we are only interested in the statistical properties of the sample and since the signal to noise in the recombination line data from this survey is lower than for any of the observations of individual sources, we set a somewhat lower threshold for BRLOs and include objects with RRL widths $\geq 45 \mathrm{~km} \mathrm{~s}^{-1}$. Using this criterion, 6 of the 23 sources in the sample have lines broad enough to demonstrate the presence of one or more BRLOs which also implies a frequency of incidence of roughly $30 \%$ for BRLOs in their sample.

Table 3 gives a summary of the main characteristics of all the well-studied BRLOs (i.e. not including the statistical sample from the Afflerbach et al. survey). Most of the sources are very compact, with typical crossing times of less than $2 \times 10^{3}$ years for expansion velocities of around $50 \mathrm{~km} \mathrm{~s}^{-1}$. Following the same line of argumentation used by Wood \& Churchwell (1989) for all compact HII regions, we find that, for such short dynamical times, one would expect to detect only $\sim 15$ objects of this kind in the whole galaxy, close to the number of objects so far detected in just a few regions. The high detection rate for BRLOs means that these objects cannot be expanding at the velocities measured by the recombination lines. 


\subsubsection{Continuum Properties}

Most of the resolved BRLOs have a bipolar morphology and almost all BRLOs have rising continuum spectra up to at least $1.3 \mathrm{~cm}$ (Table 3). Even for the most compact objects, the rising spectrum continues well into the millimeter wavelengths. Unfortunately, the complexity of most of the regions and the presence of large column densities of dust makes it very difficult to measure the continuum spectral indexes at short millimeter and sub-millimeter wavelengths. For the best case, MWC 349, the $\nu^{0.6}$ continuum spectrum is observed up to $60 \mu \mathrm{m}$ (Harvey et al. 1979). If other BRLOs share this characteristic, large emission measures lie hidden in the optically thick regions seen at centimeter wavelengths in most of the BRLOs in Table 3. As determined from the high-resolution VLA maps, all the BRLOs have emission measures of at least $10^{8} \mathrm{pc} \mathrm{cm}^{-6}$ and the number of the Lyman continuum photons needed to explain the radio data varies from $10^{47.6} \mathrm{~s}^{-1}$ for S106 to more than $10^{48.6} \mathrm{~s}^{-1}$ for the other sources. For the sources with continuum spectra that rise into the millimeter wavelengths, these values are clearly lower limits and the emission measure and the number of Lyman continuum photons increase with frequency at least with the same power law as the continuum spectrum. This indicates that the ionization of all the BRLOs, except for S106, will require stars with spectral types earlier than O6. The large emission measure indicates that the BRLOs must be ionized by a star or stars at the geometrical centers of the HII regions, within the optically thick cores observed at centimeter wavelengths. The central location of the ionizing stars is already apparent in MWC 349 and S106 where the geometry is clear because the ionizing stars are observed in the visible and the IR.

The rising continuum spectral index of an opaque ionized source is a product of a combination of the radial density and temperature distributions of the ionized material. This index is nearly independent of the axial and azimuthal geometry (Schmid-Burgk 1982). 
The BRLOs mapped with the VLA at high angular resolution show that the continuum and recombination line intensities are consistent with minor variations of the electron temperatures within the sources (see e.g De Pree et al. 1994 for K3-50, Gaume et al. 1995 for NGC 7538 IRS1; Martín-Pintado et al. 1993), suggesting that the HII regions are close to isothermal. Furthermore, for the typical spectral indexes measured for the BRLOs, between 0.5 and 1.5, the effects of moderate power law temperature gradients have minor effects $(\sim 20 \%)$ on the continuum spectral indexes; being independent of temperature gradients for spectral indexes of $\sim 0.6$ (Cassinelli \& Hartmann 1977). Therefore, we will consider that the spectral indexes given in Table 3 are basically related to the density structure within the BRLOs. The density structure varies from $n \propto \mathrm{r}^{-2}$ to $\mathrm{n} \propto \mathrm{r}^{-3}$ for spectral indexes between $\simeq 0.6$ and 1.3 respectively (Olnon 1975; Panagia \& Felli 1975; Wright \& Barlow 1975). For such steep density gradients, the HII region is far from pressure equilibrium and the ionized material must be flowing away from the dense region near the star. From mass conservation, the density structures derived from the continuum spectral indexes, imply that only in a small fraction of BRLOs (those with spectral indexes of $\sim 0.6$ ) is the ionized gas outflowing at constant velocity, and in most of the BRLOs the ionized gas is accelerating. In the most extreme case (spectral indexes of 1.3), the velocity must be proportional to the radius.

\subsubsection{Line Widths}

The measured linewidths of the BRLOs (Table 2) fall within a remarkably small range. Our best estimate for the completeness range for our data is $\Delta \mathrm{V}(\mathrm{FWHM})=45-100 \mathrm{~km} \mathrm{~s}^{-1}$. Confusion with narrow line emission from the same complex imposes the lower bound. We estimate the upper bound from our observed spectra. The upper bound results from a combination of the diminuition of peak intensity with increased width and confusion with 
the helium lines and with low-level molecular line emission. From our own observations, however, the widths of the broad recombination line components cluster strongly around 75 $\mathrm{km} \mathrm{s}^{-1}$. The results in Table 3 show that even sources observed at centimeter wavelengths with interferometers have broad recombination lines with widths very close to $75 \mathrm{~km} \mathrm{~s}^{-1}$. If further observations bear out this consistency in the velocity spread of the emission, the explanation of this result must form an important part of any theory of BRLOs.

\subsection{Possible Models}

In this section, we summarize the various possible models for UC H II regions in the context of the our new data and other recently published data on broad recombination line emission. We note first that the BRLOs are the products of gas dynamics, rather than radiative transfer. RRL observers are conditioned to think about pressure broadening whenever they see broad line profiles or wide wings on the lines. We can rule out pressure broadening, however, as the source of the wide lines in BRLOs. The very strong dependence of the pressure broadened line width on principle quantum number, $n\left(\Delta V \propto n^{7}\right)$ means that,

if pressure broadening produces the wide lines, their widths should change dramatically (by factors of 5-400) over the range of $n$ values observed for our BRLOs. In all cases for which there are observations of $\operatorname{Hn} \alpha$ transitions over a range of $\mathrm{n}$, the observed changes in the width and/or strength of the broad recombination line component are much smaller than those predicted by pressure broadening.

\subsubsection{Champagne Flows}

Champagne models (Tenorio-Tagle 1979, Bodenheimer, Tenorio-Tagle, \& Yorke 1979) consider the evolution of young H II regions as they break out of the dense neutral cores 
in which they form into a lower density diffuse medium. During the breakout phase, the ionized gas accelerates into the low density medium at speeds up to $30-50 \mathrm{~km} \mathrm{~s}^{-1}$. Subsequent analysis shows that this kind of acceleration also occurs in H II regions that form in clouds with power law density distributions, if the density gradients are sufficiently steep (Franco, Tenorio-Tagle, \& Bodenheimer 1990). A champagne phase should be a common occurence among UC H II regions, if they really form as a result of ionization of the surrounding molecular core, because of their extremely large internal pressures.

\subsubsection{Ionization of Neutral Winds}

Hypersonic bipolar outflows of molecular gas are a common feature of protostellar sources, both in low mass star forming regions and in regions containing low and high mass pre main sequence objects (Bachiller 1996; Plume et al. 1992). These outflows have velocities ranging from a few up to about $100 \mathrm{~km} \mathrm{~s}^{-1}$. External ionization of molecular flows near $\mathrm{O}$ stars embedded in a young cluster could reproduce at least some of the features of BRLOs. Alternatively, if the turn-on time for the ionizing star is short enough and occurs soon enough after the outflow phase, the O star could ionize its own molecular flow from the inside.

$\mathrm{K} 3-50 \mathrm{~A}$ is the only one of the broad line sources that is large enough $(0.2 \times 1.2 \mathrm{pc})$ to have a reasonably long crossing time $\left(\sim 2 \times 10^{4}\right.$ years $)$. In principle, then, this source would be a good candidate for the ionization of a fossil flow. In such a picture, we would expect the ionizing photons to move through the cavity evacuated by a fast molecular wind and strike the swept-up material in the cavity walls. The dense, UV-illuminated walls would appear as an elongated, limb-brightened shell. Existing continuum maps do not show such a shell (De Pree et al. 1994). 


\subsubsection{Wind-Driven Bubbles}

The fast (1000-2000 $\mathrm{km} \mathrm{s}^{-1}$ ) stellar winds from young O stars will interact strongly with the surrounding interstellar gas. After a short time $\left(\sim 10^{3}\right.$ years, Shull 1980,1982$)$, the wind will create a bubble with a dense shell of swept-up material. If this bubble forms in a flattened structure, it may result in formation of a bipolar outflow of ionized material (Koo \& McKee 1992).

\subsubsection{Mass-Loaded Winds}

Redman, Williams, \& Dyson (1995, 1996; Dyson, Williams \& Redman 1995) and Lizano et al. (1996) have developed a scheme to produce long-lasting UC H II regions in which material photoevaporated from dense circumstellar clumps loads the $\mathrm{O}$ star wind and produces a dense outflow of ionized material bounded by a recombination front.

\subsubsection{Disk Winds}

Hollenbach et al. (1994) and Yorke and collaborators (Yorke \& Welz 1993, 1996; Richling \& Yorke 1997) have suggested that evaporative flows from massive circumstellar disks may offer a solution to the lifetime problem for UC H II regions. In their models, stellar UV photons ionize material in the disk and produce steady mass loss for periods of up to $10^{5}$ years. These flows are inherently bipolar. It is possible, however, to produce asymmetric regions by invoking external photoevaporation of the disks of nearby companion stars (Kessel, Yorke, \& Richling 1998). Most of the papers discuss the terminal velocity of this evaporated material but do not explicitly model it. Kessel et al. (1998) present line profiles for optical transitions showing typical linewidths on the order of $40 \mathrm{~km} \mathrm{~s}^{-1}$. 


\subsubsection{Remarks on the Models}

Since the widespread existence of BRLOs was unknown at the time the various theoretical papers were published, the models do not explicitly address the issues raised by the nature of these sources. Nevertheless, the papers do allow us to categorize the potential problems of the various models. None of the models has an obvious explanation for the relatively narrow range in observed line widths. All but the last two models suffer from difficulties with the lifetime. They produce high velocity emission only for the order of a single crossing time and therefore cannot account for the number and frequency of observed BRLOs. We therefore consider only the mass loaded wind and photoevaporated disk models in detail.

The mass-loaded wind models can only account for the bipolar morphology of most BRLOs if the underlying protostellar wind is strongly bipolar (the photoevaporated disk models, by contrast, only work if all of the BRLOs turn out to be bipolar). Mass loaded wind models also fail to explain the $\mathrm{r}^{-2}$ or steeper density gradients inferred from the continuum spectral indices. Mass loading should result in density profiles less steep than $\mathrm{r}^{-2}$. The existing versions of the mass-loaded wind and photoevaporated disk models also do not predict the very broad recombination line profiles we observe.

On balance, the photoevaporated disk model has fewer problems than the mass-loaded wind model. There are observations that provide some supporting observational evidence for the presence of disks, if not for their involvement in the generation of the broad lines. For the best-studied BRLO, MWC 349, the hot dust surrounding the star shows a disk-like distribution with a size of $\leq 50 \times 100 \mathrm{AU}$ (Leinert 1986). A similar size for the neutral disk has also been inferred from the high angular resolution radio continuum map at $1.3 \mathrm{~cm}$ (Martín-Pintado et al. 1993). Direct evidence of the interface region between the neutral disk and the ionized gas has been measured from the $\mathrm{H} 30 \alpha$ recombination line maser spikes 
which also indicates a face-on disk of $\sim 80$ AU (Planesas, Martín-Pintado \& Serabyn 1992). The most detailed knowledge of the kinematics of the circumstellar gas comes from the IR recombination lines (Hamann \& Simon 1986) and the observations of the H30 $\alpha$ maser line. Modeling of the IR line profiles indicates the presence of a neutral rotating disk and a bipolar ionized wind. The location and the radial velocities of the H30 $\alpha$ maser spikes are consistent with a keplerian disk rotating around a $30 \mathrm{M}_{\odot}$ object (Planesas et al. 1992; Thum, Martín-Pintado \& Bachiller 1992). The presence of a disk in S106 is also inferred from the morphology of the ionized gas (see Felli et al. 1985). Unfortunately, for the other BRLOs, the properties of the disks are unknown. We note, however, that these sources are excellent candidates for the detection of recombination line masers at millimeter wavelengths, similar to those observed in MWC 349 (Martín-Pintado et al. 1989). Detection of maser emission might allow us to observe the interface region between the neutral disk and the ionized material.

\subsection{Conclusions}

1) BRLOs are common in regions containing embedded OB stars. These objects have

recombination line widths of $60-80 \mathrm{~km} \mathrm{~s}^{-1}$ and continuum spectra that rise as $\nu^{\sim 0.3}$ to $\nu^{\sim 1.0}$ into the millimeter band. Most spatially resolved BRLOs have bipolar morphology.

2) The short crossing times and high frequency of occurence of BRLOs implies both that these objects last for much longer than their expansion times and that many UCH II regions must begin their lives as BRLOs.

3) The high continuum opacity of BRLOs at short centimeter wavelengths means that estimates of dust extinction based on near-IR lines and radio continuum measurements probably overestimate the extinction. 
4) None of the published models of UCH II regions explicitly deals with all of the observed properties of BRLOs. If a photoevaporated disk is the source of the ionized bipolar emission region, the results imply that disks around massive young stars are both common and long-lived.

This work was supported in part by National Science Foundation grant 95-30695 to the University of Texas at Austin. 


\section{REFERENCES}

Acord, J.M., Churchwell, E., \& Wood, D.O.S. 1998, ApJ, 495, L107

Acord, J.M., Walmsley, C.M., \& Churchwell 1997, ApJ, 475, 693

Afflerbach, A., Churchwell, E., Acord, J.M., Hofner, P., Kurtz,S., \& De Pree, C.G. 1996, ApJS, 106, 423

Akabane, K., et al. 1992, PASJ, 44, 421

Altenhoff, W. J., Strittmatter, P. A. \& Wendker, H. J. 1981, A\&A, 93, 48

Bachiller, R. 1996, ARA\&A, 34, 111

Bally, J. \& Lada, C.J. 1983, ApJ 265, 824

Bally, J., Snell, R.L., and Predmore, R. 1983, ApJ, 272, 154

Benson, J.M., and Johnston, K.J. 1984, ApJ, 277, 181

Bodenheimer, P., Tenorio-Tagle, G., \& Yorke, H.W. 1979, ApJ, 233, 85

Bunn, J.C., Hoare, M.G., \& Drew, J.E. 1995, MNRAS, 272, 346

Campbell, B. 1984, ApJ 282, L27

Cassinelli, J.P., \& Hartmann, L. 1977, AJ, 212,488

Chlebowski, T., \& Garmany, C.D. 1991, ApJ, 368, 241

Churchwell, E., Walmsley, C.M., Wood, D.O.S., \& Steppe, H. 1989, in Radio Recombination Lines: 25 Years of Investigation, M. Gordon \& R.L. Sorochenko eds, (Kluwer: Dordrecht), 83

De Pree, C.G., Gaume, R.A., Goss, W.M., \& Claussen, M.J. 1996, ApJ, 464, 788

De Pree, C.G., Goss, W.M., Palmer, P., \& Rubin, R.H. 1994, ApJ, 428, 670

De Pree, C.G., Mehringer, D.M., \& Goss, W.M. 1997, ApJ, 482, 307 
Dreher, J.W., Johnston, K.J., Welch, W.J., and Walker, R.C. 1984, ApJ, 283, 632

Drew, J.E., Bunn, J.C., \& Hoare, M.G. 1993, MNRAS, 265, 12

Dyson, J.E., Williams, R.J.R., \& Redman, M.P. 1995, MNRAS, 277, 700

Eiroa, C., Elsaesser, H., \& Lahulla, J.F. 1979, A\&A, 74, 89

Felli, M., Simon, M., Fischer, J., and Hamann, F. 1985, A\&A145, 305

Felli, M., Staude, H.J., Reddmann, T., Massi, M., Eiroa, C., Hefele, H., Neckel, T., \& Panagia, N. 1984, A\&A, 135, 261

Franco, J., Tenorio-Tagle, G., \& Bodenheimer, P. 1990, ApJ, 349, 126

Garay, G. 1989, in Radio Recombination Lines: 25 Years of Investigation, M. Gordon \& R.L. Sorochenko eds, (Kluwer: Dordrecht), 73

Garay, G., Rodriguez, L.F., and van Gorkom, J.H. 1986, ApJ, 309, 553

Garden, R.P. and Geballe, T.R. 1986, MNRAS, 220, 611

Gaume, R.A., Fey, A.L., \& Claussen, M.J. 1994, ApJ, 432, 648

Gaume, R.A., Goss, W.M., Dickel, H.R., Wilson, T.L., \& Johnston, K.J. 1995, ApJ, 438, 776

Gordon, M.A. 1989, ApJ, 337, 782

Hamann, F. \& Simon, M. 1986, ApJ, 311, 909

Hartmann, L., Jaffe, D.T. \& Huchra, J.P. 1980, ApJ, 239, 905

Harvey, P.M., Thronson, H.A. \& Gatley, I. 1979, ApJ, 233, 115

Hasegawa,T. and Akabane,K. 1984, ApJ, 287, L91

Ho, P.T.P., \& Haschick, A.D. 1981, ApJ, 248, 622

Ho, P.T.P., \& Haschick, A.D. 1986, ApJ, 304, 501 
Hoare, M.G., Drew, J.E., Muxlow, T.B., \& Davis, R.J. 1994, ApJ, 421, L51

Hollenbach, D.J., Johnstone, D., Lizano, S., \& Shu, F. 1994, ApJ, 428, 654

Kessel, O., Yorke, H.W., \& Richling, S. 1998, A\&A, 337, 832

Koo, B.C., and McKee, C.F. 1992, ApJ, 388, 93

Leinert, C. 1986, A\&A, 155, L6

Lizano, S., Cantó, J., Garay, G., \& Hollenbach, D.J. 1996, ApJ, 468, 739

Martín-Pintado, J., Bachiller, R., Thum, C., and Walmsley, C.M. 1989, A\&A, 215, L13

Martín-Pintado, J., Gaume, R., Bachiller, R., Johnston, K., Planesas, P., 1993, ApJL, 418, L79

McKee, C.F., and Hollenbach, D.J. 1987, ApJ, 322, 275

Meaburn, J., and Walsh, J.R. 1981, Ap. and Spac. Sci., 74, 169

Meaburn, J. and Walsh, J.R. 1986, MNRAS, 220, 745

Mehringer, D.M., De Pree, C.G., Gaume, R.A., Goss, W.M., \& Claussen, M.J. 1995, ApJ, 442, L29

Olnon, F.M. 1975, A\&A, 39, 127

Panagia, N., and Felli, M. 1975, A\&A, 39, 1

Persson, S.E., Geballe, T.R., McGregor, P.J., Edwards, S., and Lonsdale, C.J. 1984, ApJ, 286, 289

Planesas, P., Martin-Pintado, J., \& Serabyn, E. 1992, ApJL, 386, 23

Plume, R., Jaffe, D.T., \& Evans, N.J. II 1992, ApJS, 78, 50

Plume, R., Jaffe, D.T., Evans, N.J. II, Martin-Pintado, J., \& Gomez-Gonzalez, J. 1997, ApJ, 476, 730

Redman, M.P., Williams, R., \& Dyson, J.E. 1995, Ap. \& Spac. Sci., 233, 195 
Redman, M.P., Williams, R., \& Dyson, J.E. 1996, MNRAS, 280, 661

Reid, M.J., and Ho, P.T.P. 1985, ApJ, 28, L17

Richer, J.S., Padman, R., Ward-Thompson, D., Hills, R.E., \& Harris, A.I. 1993, MNRAS, 262,839

Richling, S., \& Yorke, H.W. 1997, A\&A, 327, 317

Roelfsma, P.R., Goss, W.M., \& Geballe, T.R. 1988, A\&A, 207, 132

Russell, A.P.G., Bally, J., Padman, R., and Hills, R.E. 1992, ApJ, 387, 219

Schmid-Burgk, J., 1982, A\&A, 108, 169

Serabyn, E., Guesten, R., and Schulz, A. 1993, ApJ, 413, 571

Shull, J.M. 1980, ApJ, 238, 860

Shull, J.M. 1982, in "Regions of Recent Star Formation", R.S. Roger and P.E. Dewdney eds., (Dordrecht; Reidel), p. 91

Simon, M., Felli, M., Cassar, L., Fischer, J., and Massi, M. 1983, ApJ, 266, 623

Simon, M., Righini-Cohen, G., Fischer, J., and Cassar, L. 1981, ApJ, 251, 552

Snell, R.L., \& Bally, J. 1986, ApJ 303, 683

Solf, J., \& Carsenty, U. 1982, A\&A, 113, 142

Tenorio-Tagle, G. 1979, A\&A, 71, 59

Thum, C. Martín-Pintado, J., Bachiller, R. 1992, A\&A, 256, 507 E. 1990, ApJ, 353, 570

White, R.L. \& Becker, R.H. 1985, ApJ, 297, 677

Wood, D.O.S., \& Churchwell, E. 1989, ApJS, 69, 831

Wright, A.E., \& Barlow, M.J. 1975, MNRAS, 170, 41

Yorke, H.W., \& Welz, A. 1993, in "Star Formation, Galaxies, and the Interstellar Medium", J. Franco, F. Ferrini, and G. Tenorio-Tagle eds., (Cambridge), p. 239. 
Yorke, H.W., \& Welz, A. 1996, A\&A, 315, 555

Zijlstra, A.A., Pottasch, S.R., Engels, D., Roelfsma, P.R., te Lintel Hekkert, P., \& Umana, G. 1990, MNRAS, 246, 217 


\section{Figure Captions}

Fig. 1. - Comparison of fits of one and two Gaussians to the H41 $\alpha$ line toward NGC 7538 IRS1. (left) Single Gaussian fit to the region within $\pm 15 \mathrm{~km} \mathrm{~s}^{-1}$ of the peak intensity and residuals to that fit. (right) Two Gaussian fit to the same profile (line parameters in Table 2). Most of the power in the residuals to this fit is due to either $\mathrm{He} 41 \alpha$ or to the molecular feature seen at higher velocities $\left(\mathrm{CH}_{3} \mathrm{CN}\right)$.

Fig. 2.- Comparison of fits of one and two Gaussians to the H41 $\alpha$ line toward W49N. (upper left) Single Gaussian fit to the region within $\pm 15 \mathrm{~km} \mathrm{~s}^{-1}$ of the peak intensity. (lower left) Residuals to the single Gaussian fit. (upper right) Two Gaussian fit to the same profile (see Table 2). (lower right) Residuals to the two Gaussian fit. The solid line shows a two Gaussian fit to the He41 $\alpha$ line made using the widths of the hydrogen features and a constant velocity offset of $-122.2 \mathrm{~km} \mathrm{~s}^{-1}$.

Fig. 3. - Comparison of $\mathrm{H} 39 \alpha$ (thin histogram) and $\mathrm{H} 41 \alpha$ (dashed histogram) profiles in W49N. (upper panel) The dotted line shows the third feature in a three Gaussian fit to H39 $\alpha$. For this fit, the width and position of the other two components were fixed to the values derived from a fit of two Gaussians to the H41 $\alpha$ profile. (lower panel) Overlay of the H39 $\alpha$ profile and an H41 $\alpha$ profile to which the third component has been artificially added.

Fig. 4. - Comparison of fits of one and two Gaussians to the H41 $\alpha$ line toward G34.3+0.2. (upper left) Single Gaussian fit to the region within $\pm 15 \mathrm{~km} \mathrm{~s}^{-1}$ of the peak intensity. (lower left) Residuals to the single Gaussian fit. (upper right) Two Gaussian fit. (lower right) Residuals to the two Gaussian fit and a two Gaussian fit to the He41 $\alpha$ line (see text).

Fig. 5.- Overlay of the H39 $\alpha$ (solid line) and H41 $\alpha$ (dashed line) spectra toward G34.3+0.2. The dashed line shows the sum of two Gaussians representing a compromise between fits to the two hydrogen transitions $\left(\mathrm{V}_{N}=53.0 \mathrm{~km} \mathrm{~s}^{-1}, \Delta \mathrm{V}_{N}=32.3 \mathrm{~km} \mathrm{~s}^{-1} ; \mathrm{V}_{B}=41.6 \mathrm{~km} \mathrm{~s}^{-} 1\right.$, 
$\left.\Delta \mathrm{V}_{B}=75.0 \mathrm{~km} \mathrm{~s}^{-1}\right)$.

Fig. 6.- (left) $\mathrm{H} 39 \alpha$ toward Orion $\mathrm{H}_{2}$ Peak 1. The smooth curve shows a single Gaussian fit to the observed spectrum (see Table 2). The residuals to this fit are below the profile. (right) H41 $\alpha$ toward Orion IRc2.

Fig. 7.- Recombination line spectra toward G10.6-0.4. (top) H41 $\alpha$. The smooth curve shows a single Gaussian fit to the hydrogen line and a fit for the intensity of the He41 $\alpha$ line with the width of this line fixed to the hydrogen line width and the velocity fixed to -122.2 $\mathrm{km} \mathrm{s}^{-1}$ with respect to the hydrogen line. (bottom) Fit to the H35 $\alpha$ line and He35 $\alpha$ line.

Fig. 8. - Single Gaussian fit to the H39 $\alpha$ line toward DR21. The He39 $\alpha$ line was fit holding the width equal to the $\mathrm{H} 39 \alpha$ width and the velocity at $-122.2 \mathrm{~km} \mathrm{~s}^{-1}$ with respect to the hydrogen line. 
Table 1. Observing Log

\begin{tabular}{|c|c|c|c|c|}
\hline Source & R.A.(1950) & Decl.(1950) & Date & Lines \\
\hline Orion $\mathrm{H}_{2}$ Peak 1 & 053246.3 & -052354 & $2 / 92$ & $\mathrm{H} 30 \alpha, \mathrm{H} 36 \alpha, \mathrm{H} 39 \alpha$ \\
\hline Orion IRc2 & 053247.0 & -052424 & $2 / 92$ & $\mathrm{H} 36 \alpha, \mathrm{H} 41 \alpha$ \\
\hline G10.6-0.4 & 180730.6 & -195628 & $3 / 93$ & $\mathrm{H} 35 \alpha, \mathrm{H} 41 \alpha$ \\
\hline \multirow[t]{2}{*}{ G34.3+0.2 } & 185046.1 & 11110 & $2 / 92$ & $\mathrm{H} 36 \alpha, \mathrm{H} 39 \alpha, \mathrm{H} 41 \alpha$ \\
\hline & & & $3 / 93$ & $\mathrm{H} 30 \alpha, \mathrm{H} 31 \alpha, \mathrm{H} 35 \alpha, \mathrm{H} 39 \alpha, \mathrm{H} 41 \alpha$ \\
\hline \multirow[t]{2}{*}{$\mathrm{W} 49 \mathrm{~N}$} & 190749.8 & 90117 & $2 / 92$ & $\mathrm{H} 36 \alpha, \mathrm{H} 39 \alpha, \mathrm{H} 41 \alpha$ \\
\hline & & & $3 / 93$ & $\mathrm{H} 30 \alpha, \mathrm{H} 31 \alpha, \mathrm{H} 35 \alpha, \mathrm{H} 41 \alpha$ \\
\hline \multirow[t]{2}{*}{ S106 } & 202532.8 & 371244 & $2 / 92$ & $\mathrm{H} 36 \alpha, \mathrm{H} 39 \alpha$ \\
\hline & & & $3 / 93$ & $\mathrm{H} 35 \alpha, \mathrm{H} 41 \alpha$ \\
\hline \multirow[t]{2}{*}{ DR21 } & 203714.1 & 420855 & $2 / 92$ & $\mathrm{H} 30 \alpha, \mathrm{H} 36 \alpha, \mathrm{H} 39 \alpha$ \\
\hline & 203714.2 & 420906 & $3 / 93$ & $\mathrm{H} 35 \alpha, \mathrm{H} 41 \alpha$ \\
\hline \multirow[t]{2}{*}{ NGC 7538} & 231136.5 & 611149 & $2 / 92$ & $\mathrm{H} 30 \alpha, \mathrm{H} 36 \alpha, \mathrm{H} 39 \alpha$ \\
\hline & & & $3 / 93$ & $\mathrm{H} 31 \alpha, \mathrm{H} 35 \alpha, \mathrm{H} 39 \alpha, \mathrm{H} 41 \alpha$ \\
\hline
\end{tabular}


Table 2. Continuum and recombination Line Parameters

\begin{tabular}{|c|c|c|c|c|c|c|c|c|}
\hline \multirow[t]{2}{*}{ Source } & \multirow{2}{*}{\multicolumn{2}{|c|}{ Line }} & $\mathrm{T}_{L}$ & V & $\Delta \mathrm{V}$ & $\int \mathrm{TdV}$ & $\mathrm{S}_{\nu}{ }^{\mathrm{a})}$ & $\mathrm{T}_{e}^{* \mathrm{~b})}$ \\
\hline & & & $\mathrm{K}$ & $\mathrm{km} \mathrm{s}^{-1}$ & $\mathrm{~km} \mathrm{~s}^{-1}$ & $\mathrm{~K} \mathrm{~km} \mathrm{~s}^{-1}$ & Jy & $\mathrm{K}$ \\
\hline Orion $\mathrm{H}_{2}$ Peak 1 & $\mathrm{H} 39 \alpha$ & & 0.27 & -4.34 & 25.6 & $7.3(0.25)$ & & \\
\hline Orion IRc2 & $\mathrm{H} 41 \alpha$ & & 0.54 & -3.57 & 22.7 & $13.0(0.3)$ & & \\
\hline G10.6-0.4 & $\mathrm{H} 41 \alpha$ & & 0.67 & $0.2(0.1)$ & $28.0(0.1)$ & $20.5(0.15)$ & 4.0 & 9300 \\
\hline \multirow[t]{2}{*}{$\mathrm{G} 34.3+0.2$} & $\mathrm{H} 41 \alpha$ & $\mathrm{N}$ & 0.45 & 53.0 & 32.3 & $14.9(0.4)$ & 3.0 & 9500 \\
\hline & & $\mathrm{B}$ & 0.25 & 41.6 & 75.0 & $21.1(0.6)$ & 4.2 & 9500 \\
\hline \multirow[t]{2}{*}{$\mathrm{W} 49 \mathrm{~N}$} & $\mathrm{H} 41 \alpha$ & $\mathrm{N}$ & 0.77 & 7.7 & 30.6 & 25.1 & 6.0 & 10500 \\
\hline & & $\mathrm{B}$ & 0.18 & 12.7 & 70.8 & 13.3 & 3.0 & 10500 \\
\hline \multirow[t]{2}{*}{ S106 } & $\mathrm{H} 41 \alpha$ & $\mathrm{N}$ & 0.136 & $-3.1(0.6)$ & $29.7(2.3)$ & $4.3(0.7)$ & 1.0 & 10000 \\
\hline & & $\mathrm{B}$ & 0.066 & $-1.6(2.2)$ & $75(8)$ & $5.2(0.8)$ & 1.1 & 10000 \\
\hline DR21 & $\mathrm{H} 41 \alpha$ & & 1.24 & $-0.84(0.06)$ & $32.6(0.1)$ & $43.2(0.2)$ & 10.6 & 11000 \\
\hline \multirow[t]{4}{*}{ NGC 7538} & $\mathrm{H} 41 \alpha$ & $\mathrm{N}$ & 0.094 & $-65.6(0.7)$ & $24.5(1.0)$ & $2.7(0.3)$ & 0.8 & 12800 \\
\hline & & $\mathrm{B}$ & 0.077 & $-47.8(1.9)$ & $69.3(3.0)$ & $5.8(0.4)$ & 1.7 & 12800 \\
\hline & $\mathrm{H} 39 \alpha$ & $\mathrm{N}$ & 0.095 & $-65.6(0.6)$ & $26.8(1.9)$ & $2.7(0.3)$ & & \\
\hline & & B & 0.066 & $-46.8(2.1)$ & $77.1(3.9)$ & $5.4(0.3)$ & & \\
\hline
\end{tabular}

aThe continuum flux densities for the normal (N) and broad (B) spectral components have been derived from the electron temperatures and the integrated line intensities

${ }^{\mathrm{b}}$ LTE electron temperature derived from the ratio between the total integrated line intensity $(\mathrm{B}+\mathrm{N})$ and the measured continuum emission 
Table 3. Summary of the properties of the broad recombination line sources

\begin{tabular}{ccccccc}
\hline \hline Source & Morphology & $\begin{array}{c}\text { Spectral } \\
\text { index }\end{array}$ & $\begin{array}{c}\text { Size } \\
\mathrm{pc}\end{array}$ & $\begin{array}{c}\Delta \mathrm{V} \\
\mathrm{kms}^{-1}\end{array}$ & $\begin{array}{c}\text { Cross. time } \\
10^{2} \mathrm{yr}\end{array}$ & $\begin{array}{c}\text { References to } \\
\text { col. 2 to } 5\end{array}$ \\
\hline Sgr B2F* & Compact & $\sim 1.5$ & 0.011 & 59 & 2 & $\mathrm{a}$ \\
G5.89-0.39 & Shell & $0.6,2.2$ & 0.044 & 64 & 6 & $\mathrm{~b}, \mathrm{c}, \mathrm{d}$ \\
G34.3+0.2C & Cometary & $0.9,-0.1$ & 0.035 & 75 & 5 & $\mathrm{~b}, \mathrm{e}$ \\
W49N & Unresolved & $0.6,0.9$ & 0.07 & 71 & 10 & $\mathrm{f}, \mathrm{e}$ \\
K3-50A & Bipolar & 0.5 & $0.2 \times 1.2$ & 66 & $30-180$ & $\mathrm{~g}$ \\
S106 & Bipolar & $0.73,-0.1$ & $0.07 \times 0.2$ & 75 & $9-12$ & $\mathrm{~h}, \mathrm{e}$ \\
MWC349 & Bipolar & 0.65 & $0.001 \times 0.03$ & 60 & $0.2-0.6$ & $\mathrm{i}, \mathrm{j}, \mathrm{k}$ \\
NGC7538 IRS1 & Bipolar & 0.9 & $0.003 \times 0.02$ & 64 & $0.5-3$ & $\mathrm{l}, \mathrm{e}$ \\
\hline
\end{tabular}

* 4 sources, a) De Pree et al. (1996), b) Wood \& Churchwell (1989), c) See text, d) Zijlstra et al. (1990), e) this paper, f) De Pree et al. (1996), g) De Pree et al. (1994), h) Bally, Snell \& Predmore (1983), i) Martín-Pintado et al. (1993), j) Harvey, Thronson \& Gatley (1979), k) Hartmann, Jaffe \& Huchra, (1980), l) Gaume et al. 1995) 


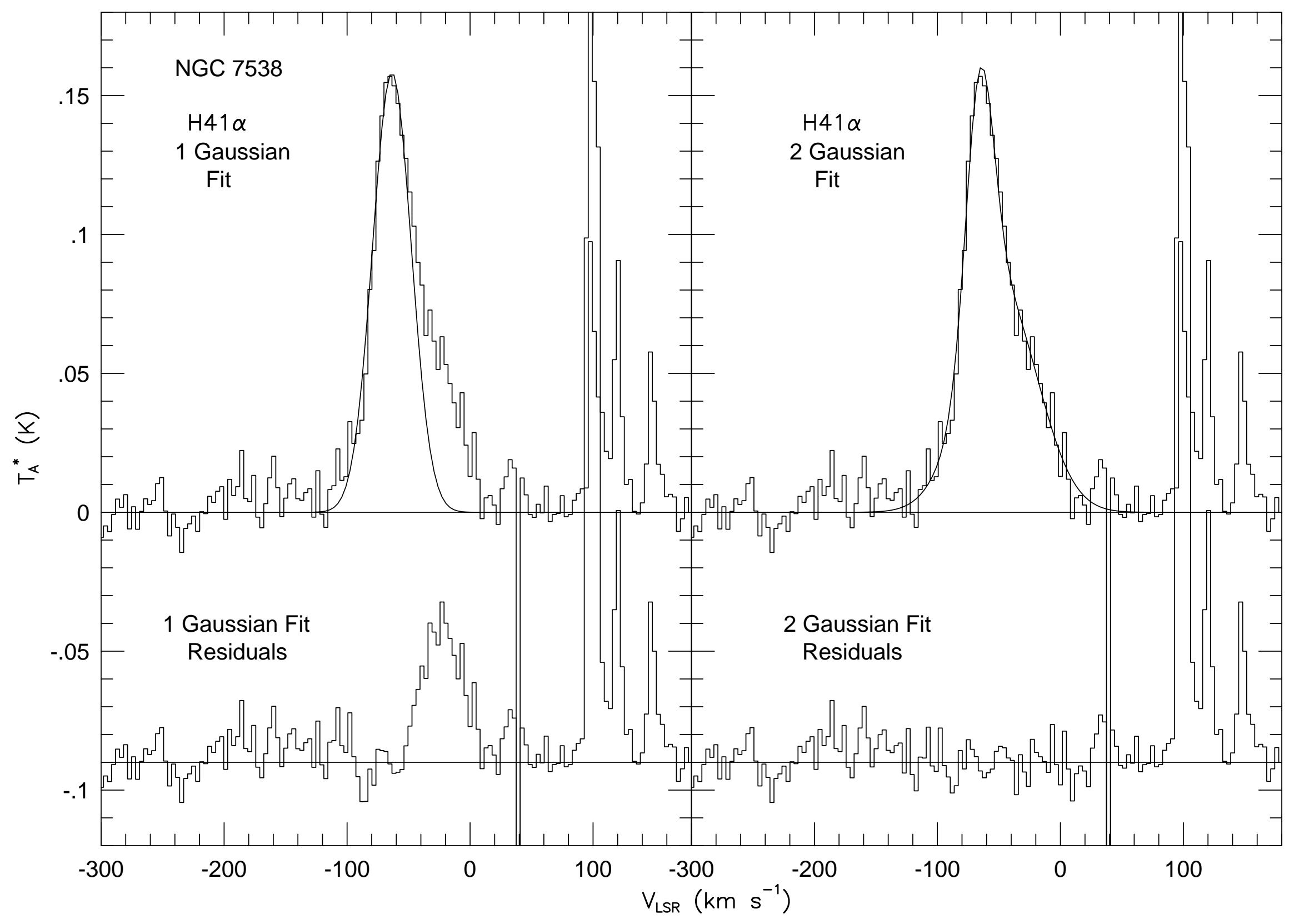




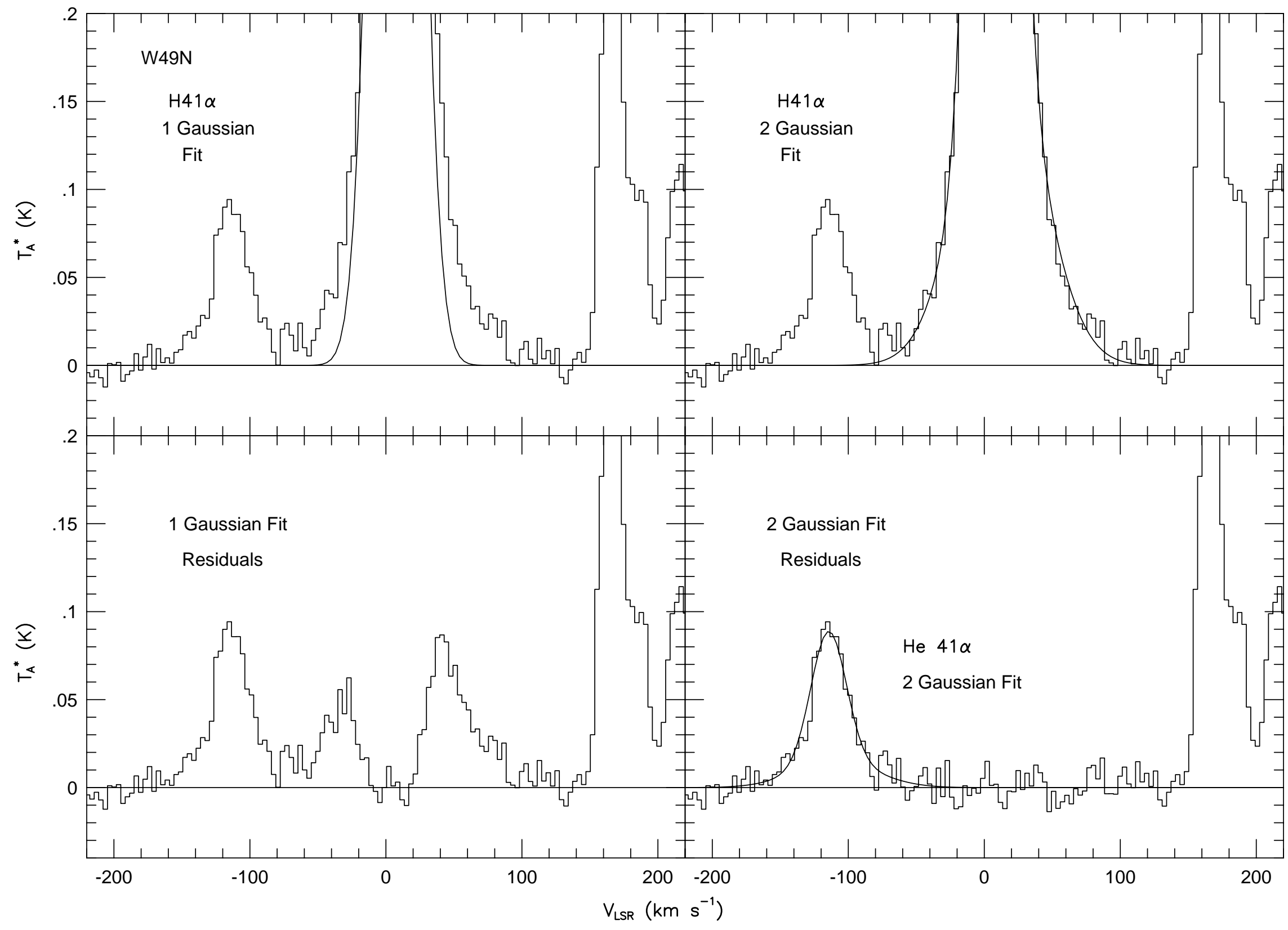




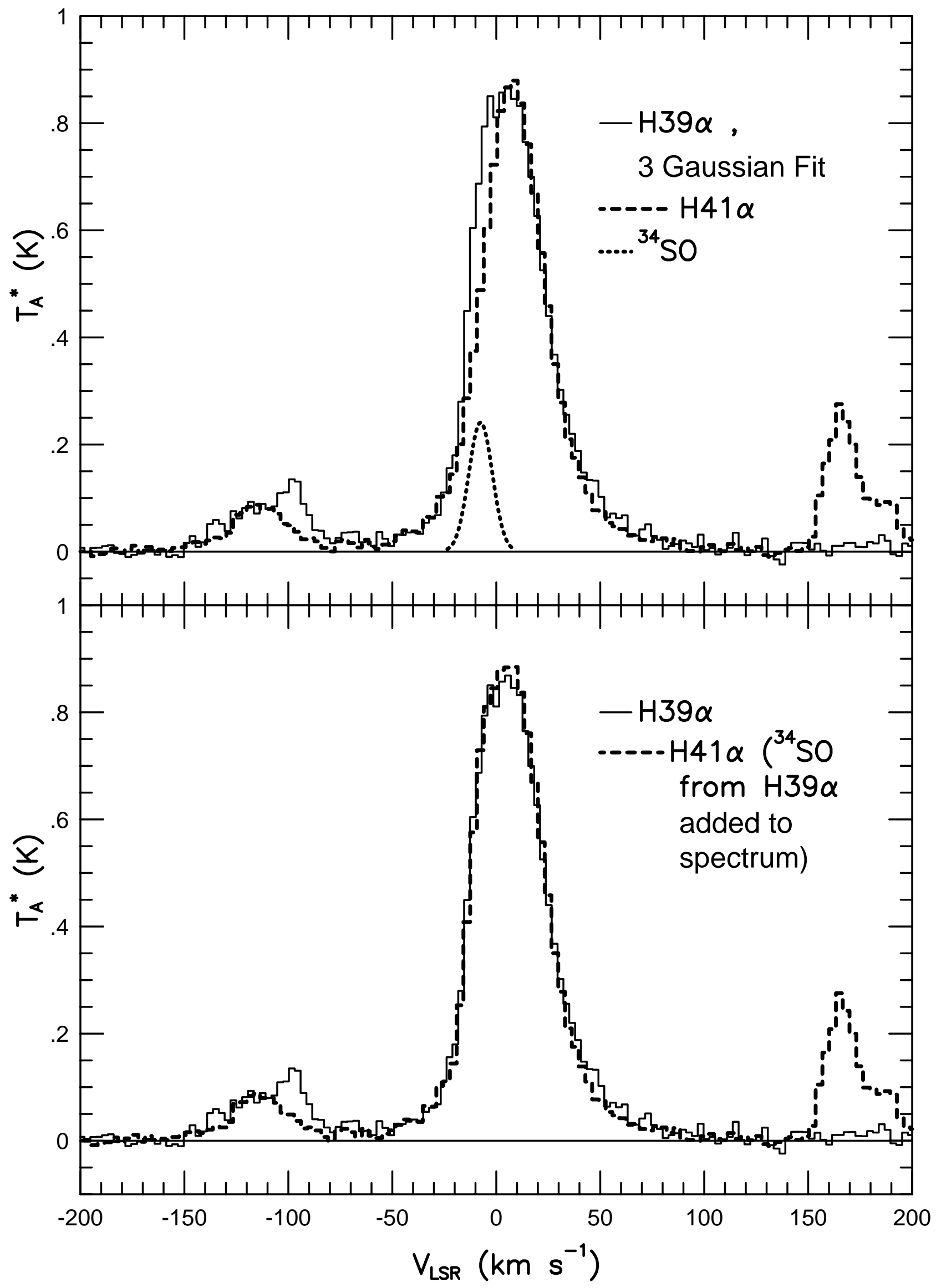




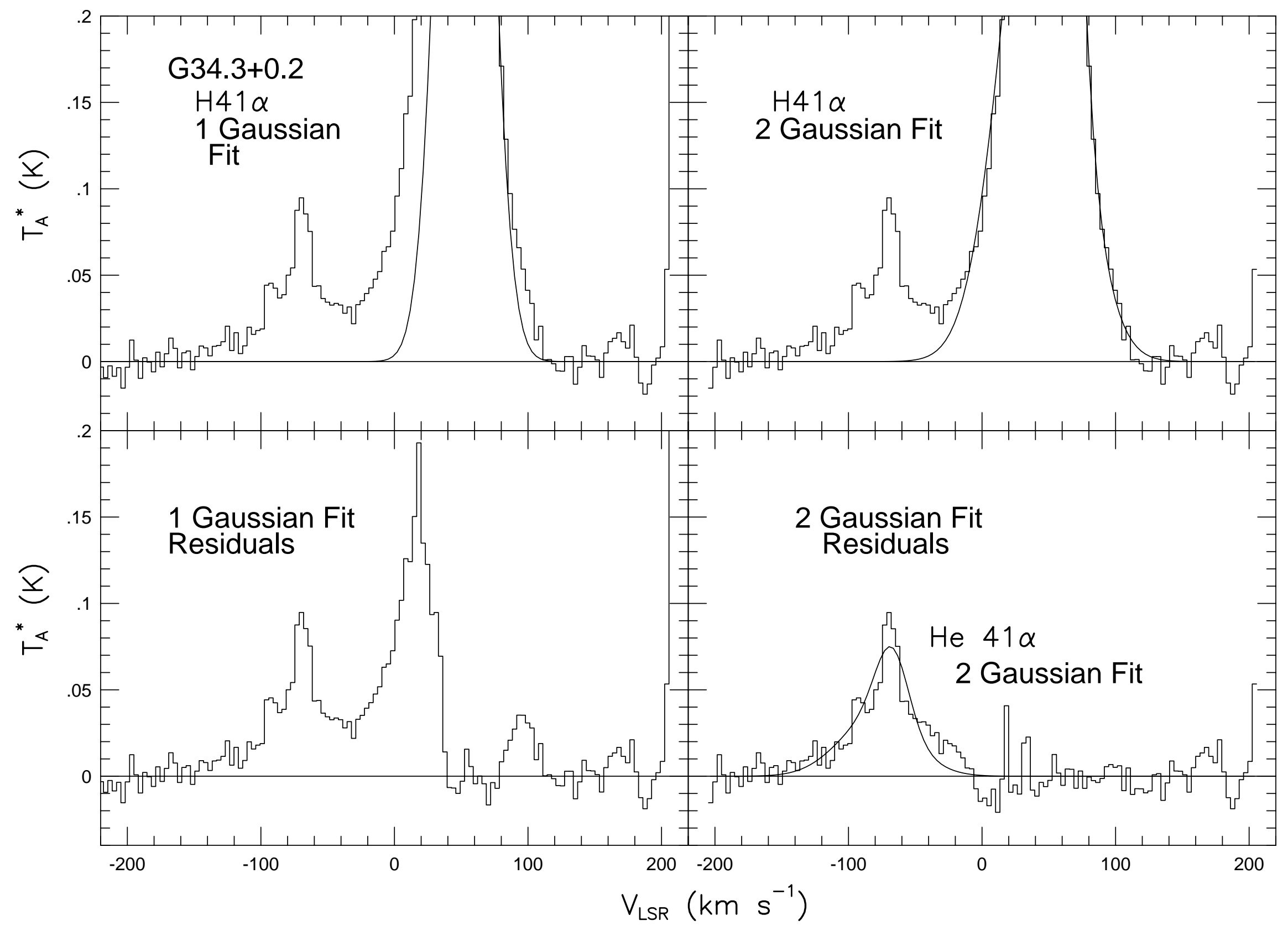




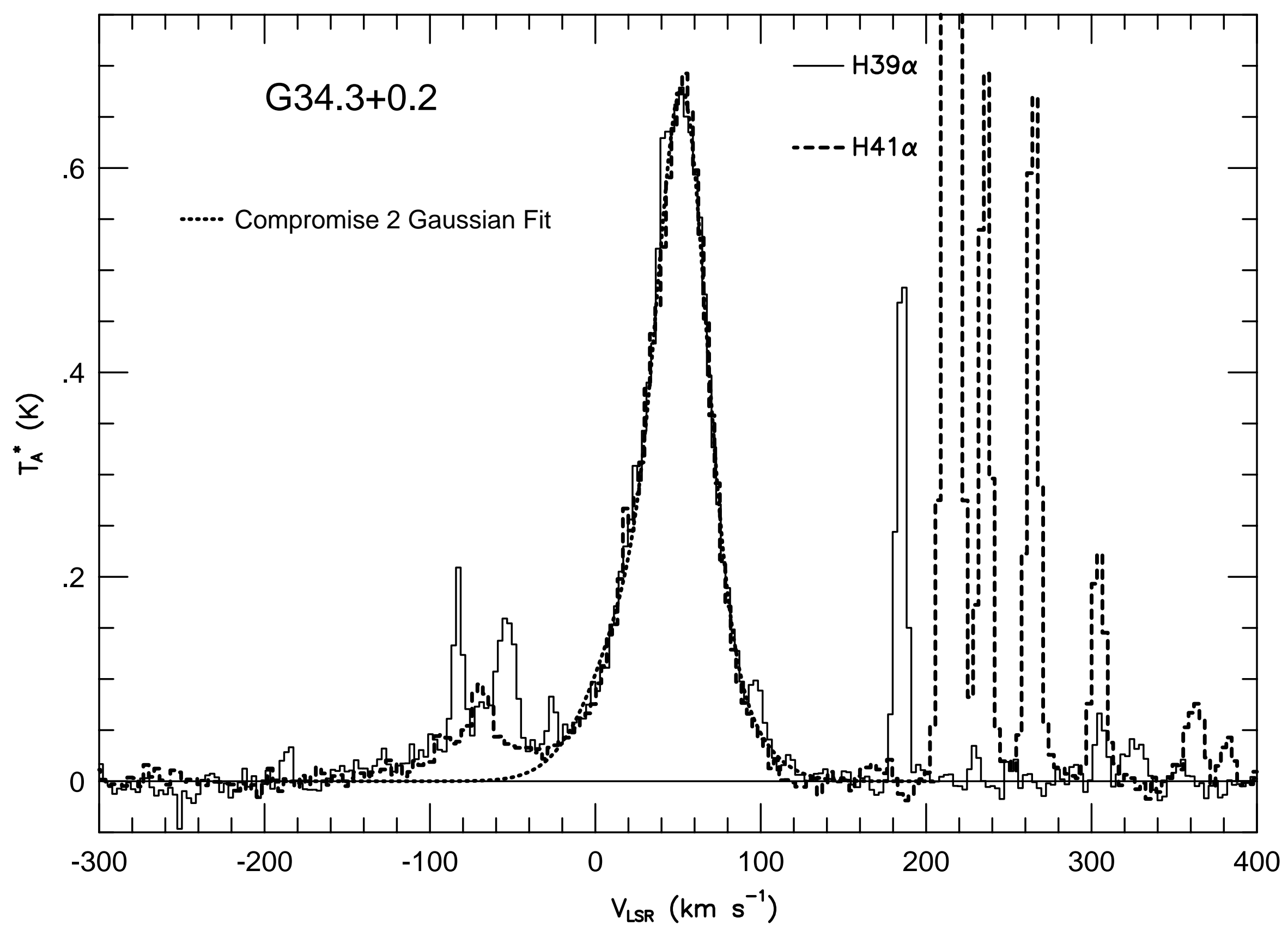



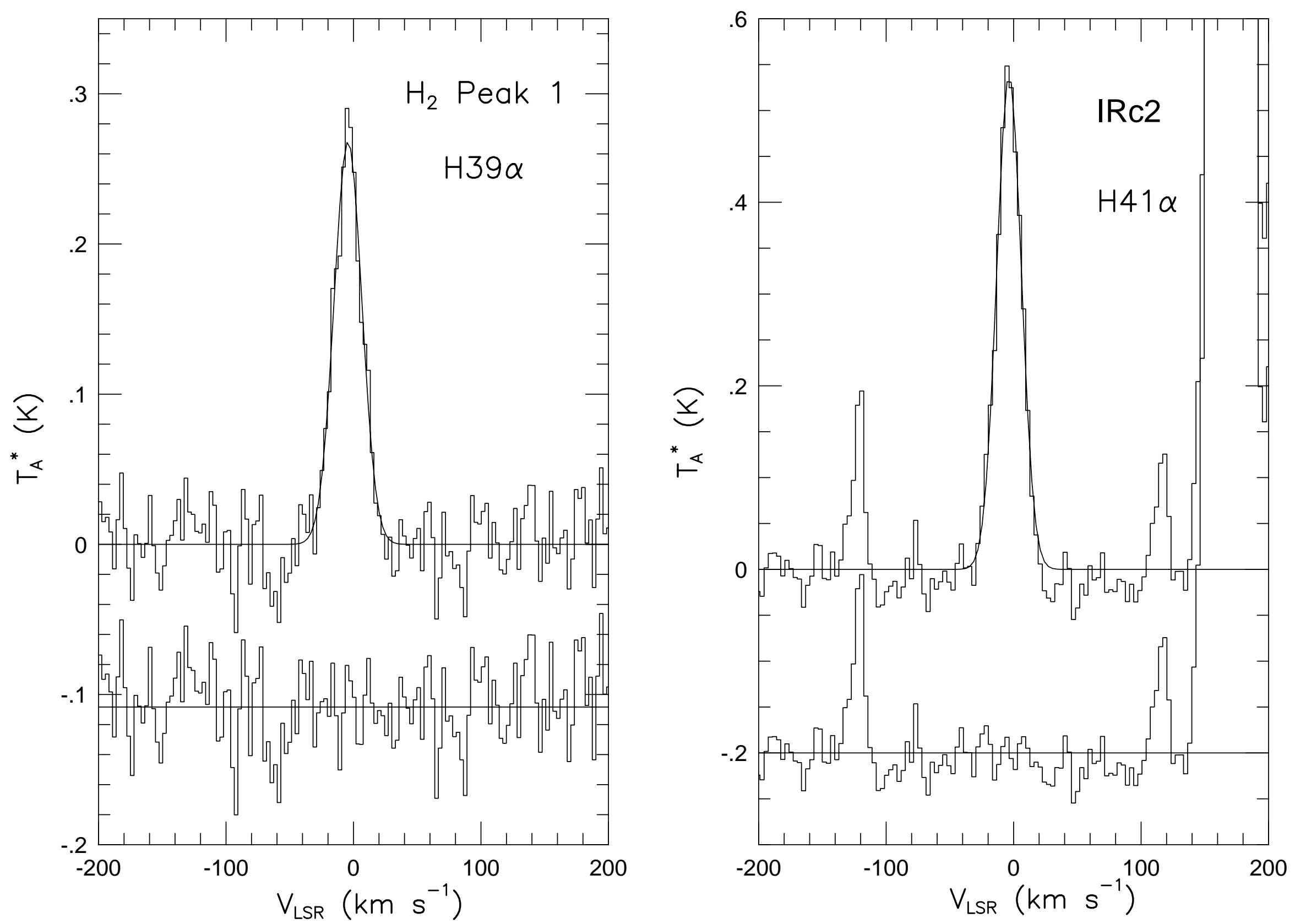


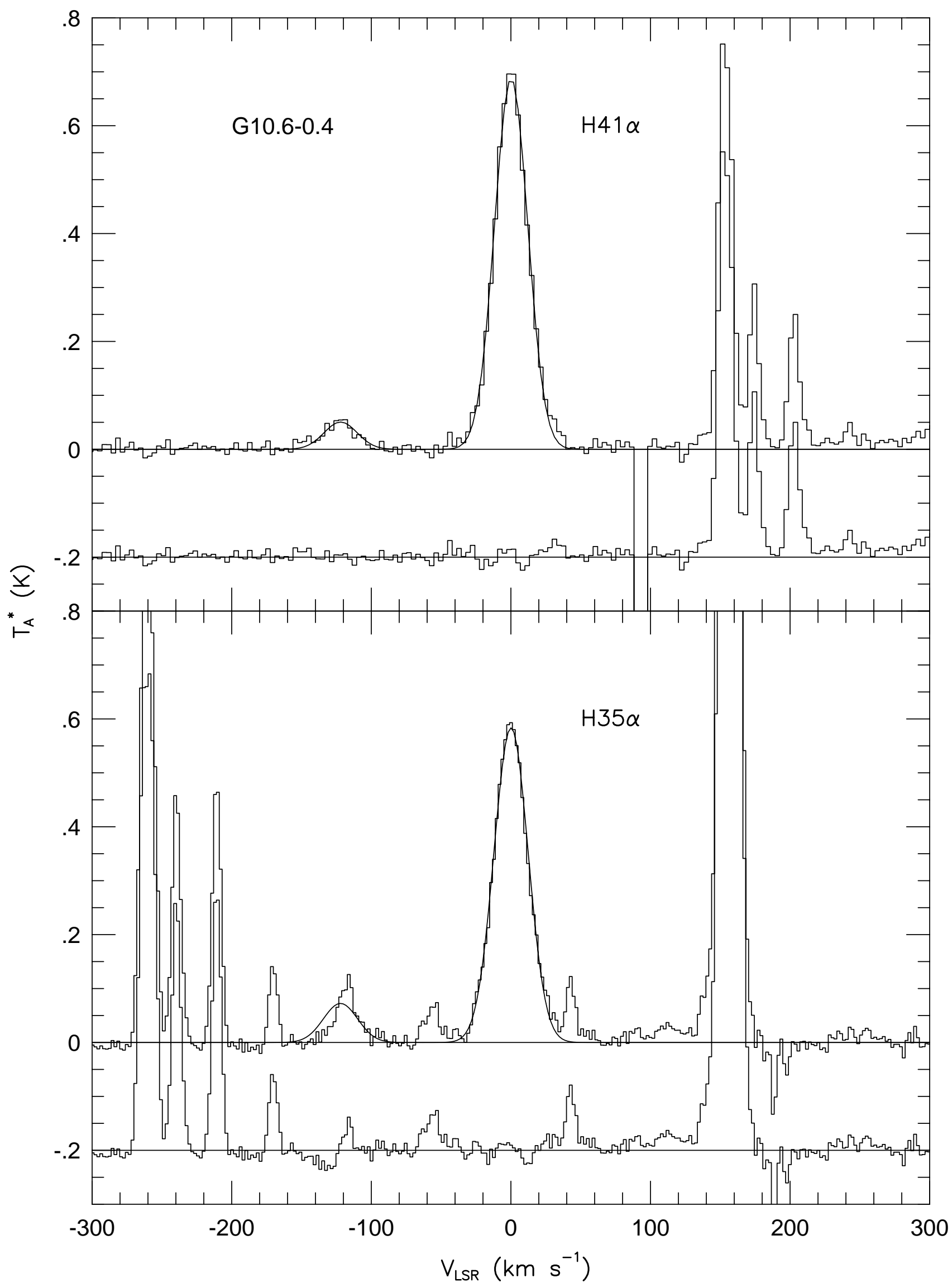




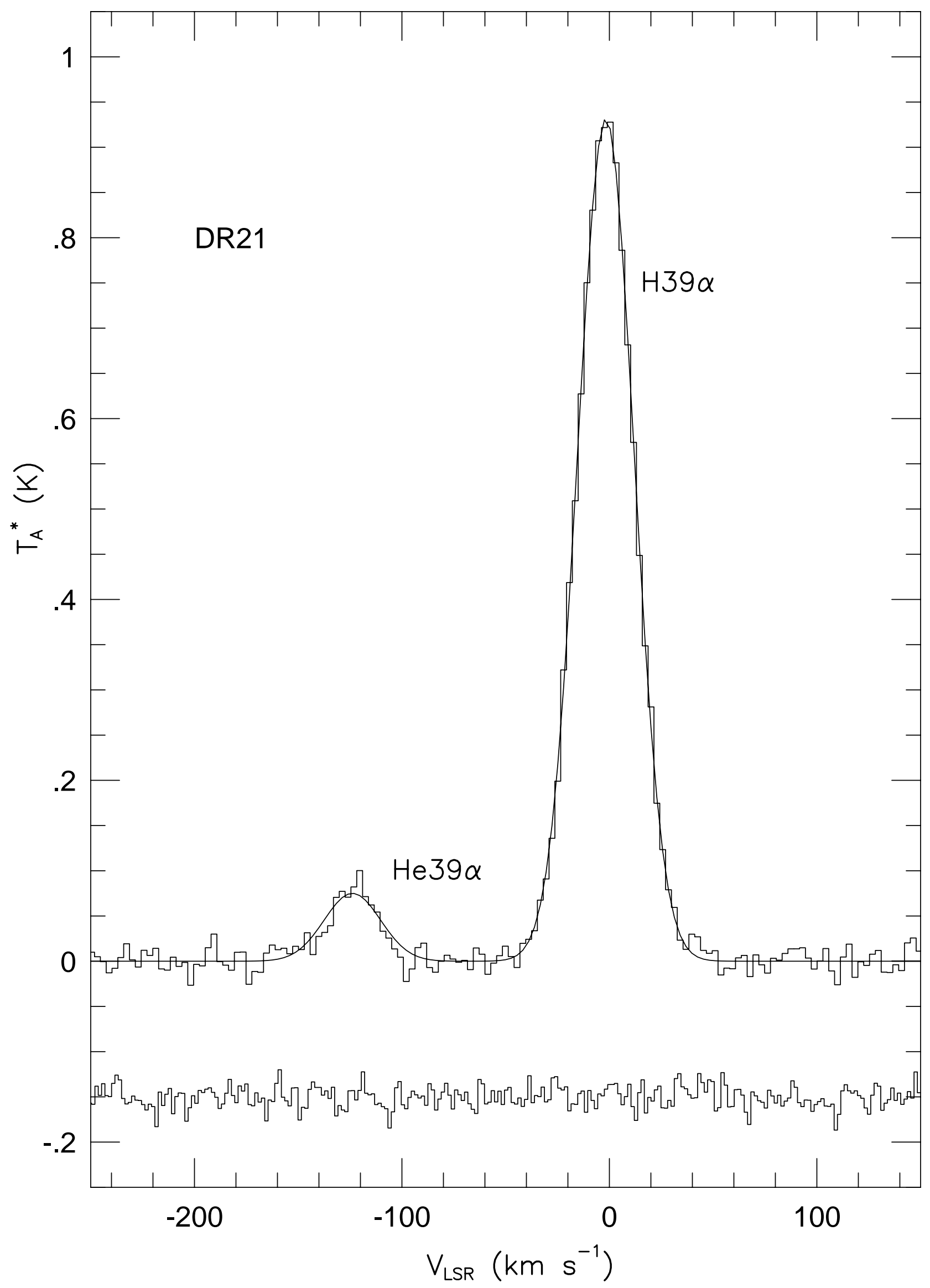

